\title{
FAÀ DI BRUNO'S FORMULA AND NONHYPERBOLIC FIXED POINTS OF ONE-DIMENSIONAL MAPS
}

\author{
VADIM PONOMARENKO
}

Received 22 June 2003

\begin{abstract}
Fixed-point theory of one-dimensional maps of $\mathbb{R}$ does not completely address the issue of nonhyperbolic fixed points. This note generalizes the existing tests to completely classify all such fixed points. To do this, a family of operators are exhibited that are analogous to generalizations of the Schwarzian derivative. In addition, a family of functions $f$ are exhibited such that the Maclaurin series of $f(f(x))$ and $x$ are identical.
\end{abstract}

2000 Mathematics Subject Classification: 37E05, 26A18, 39B12, 26A30.

1. Introduction. The study of dynamics of maps from $\mathbb{R}$ to $\mathbb{R}$ is central to many fields including discrete dynamical systems [1, 3, 5, 12], difference equations [4, 7, 9, 10], and differential equations via Poincaré [11] and Lorenz maps [1, 5, 8]. It is well known that a fixed point of such a map can be of three types. A stable fixed point attracts nearby points towards it, under iteration. An unstable fixed point repels nearby points, whereas a semistable fixed point attracts nearby points on one side (say, to the left), and repels nearby points on the other side.

Until recently, the classification of fixed points has been incomplete. Specifically, no test existed if $f\left(x^{*}\right)=x^{*}, f^{\prime}\left(x^{*}\right)=1, f^{\prime \prime}\left(x^{*}\right)=0$, and $f^{\prime \prime \prime}\left(x^{*}\right)=0$. Also, no test existed if $f\left(x^{*}\right)=x^{*}, f^{\prime}\left(x^{*}\right)=-1, S f\left(x^{*}\right)=0$, where $S f(x)$ is the Schwarzian derivative, defined in this case as $S f(x)=-f^{\prime \prime \prime}\left(x^{*}\right)-1.5\left(f^{\prime \prime}\left(x^{*}\right)\right)^{2}$. This situation was recently remedied in [2], which demonstrated a sequence of tests that fill both gaps. However, the tests for the second gap have certain inadequacies. This note restates and improves on the solution for the second gap. This is summarized in Figure 1.1.

The gray area to the lower left is from [2], and the gray boxed area marked "NEW" is from this note.

2. Summary of previously known results. Let $f: \mathbb{R} \rightarrow \mathbb{R}$ be a continuous map, and $x^{*}$ such that $f\left(x^{*}\right)=x^{*}$. We say that $x^{*}$ is semistable from the left (resp., right) if, given $\epsilon>0$, there is $\delta>0$ such that $x^{*}-\delta<x<x^{*}$ (resp., $x^{*}<x<x^{*}+\delta$ ) implies that $\left|f(f(\cdots(f(x)) \cdots))-x^{*}\right|<\epsilon$, for any positive number of iterations of $f$. If $x^{*}$ is semistable from both sides, we say that $x^{*}$ is stable, whereas if it is semistable from neither side, we say that $x^{*}$ is unstable. In the sequel, when we say a fixed point is semistable, we imply that it is not stable.

THEOREM 2.1 [2]. Let $f: \mathbb{R} \rightarrow \mathbb{R}$ be continuous with $f\left(x^{*}\right)=x^{*}$ and $f^{\prime}\left(x^{*}\right)=1$. Let $k>1$ be minimal such that $f^{(k)}\left(x^{*}\right)=A \neq 0$. Then $x^{*}$ is classified as follows. 


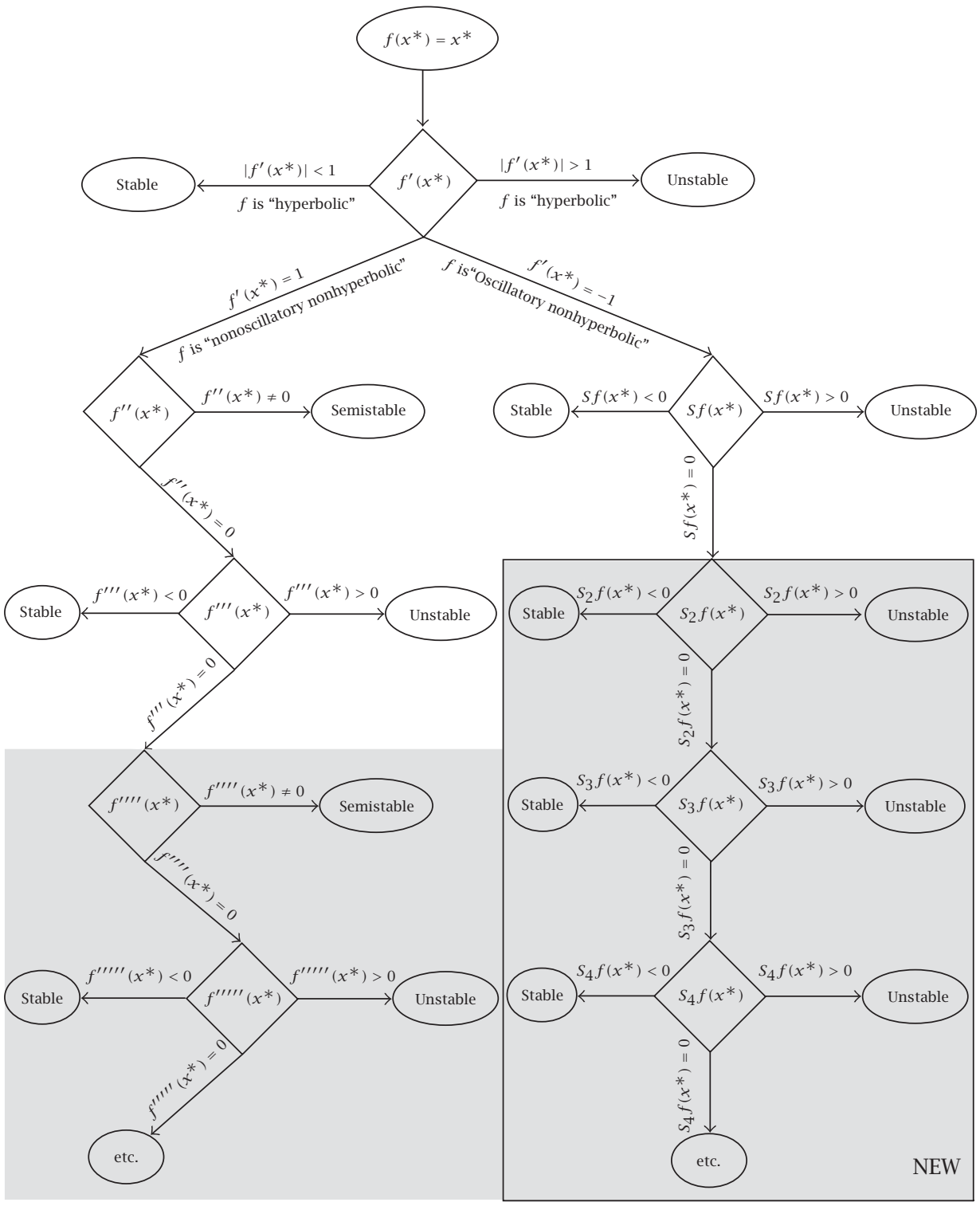

FIGURE 1.1. Classification of fixed points of a one-dimensional map.

(1) If $k$ is even and $A>0$, then $x^{*}$ is semistable from the left.

(2) If $k$ is even and $A<0$, then $x^{*}$ is semistable from the right.

(3) If $k$ is odd and $A>0$, then $x^{*}$ is unstable.

(4) If $k$ is odd and $A<0$, then $x^{*}$ is stable.

This classification of nonoscillatory nonhyperbolic fixed points (i.e., where $f^{\prime}\left(x^{*}\right)=$ 1 ) was used in [2] to generate a test for oscillatory nonhyperbolic fixed points (where 
$f^{\prime}\left(x^{*}\right)=-1$, which satisfy $S f\left(x^{*}\right)=0$ and therefore were previously unclassified. This test is due to the following classical theorem.

THEOREM 2.2. Let $f: \mathbb{R} \rightarrow \mathbb{R}$ be continuous with $f\left(x^{*}\right)=x^{*}$, and $f^{\prime}\left(x^{*}\right)=-1$. Set $g(x)=f(f(x))$. Then $x^{*}$ is classified under $f$ in the same way as under $g$.

Observe that for oscillatory nonhyperbolic fixed points, $g\left(x^{*}\right)=f\left(f\left(x^{*}\right)\right)=f\left(x^{*}\right)=$ $x^{*}$, and that $g^{\prime}\left(x^{*}\right)=f^{\prime}\left(f\left(x^{*}\right)\right) f^{\prime}\left(x^{*}\right)=f^{\prime}\left(x^{*}\right) f^{\prime}\left(x^{*}\right)=(-1)(-1)=1$. This allows us to classify $g$ (and, hence, $f$ ) using Theorem 2.1. Furthermore, [2] contains the following result, proved using Taylor's theorem.

TheOREM 2.3 [2]. Let $f: \mathbb{R} \rightarrow \mathbb{R}$ be continuous with $f\left(x^{*}\right)=x^{*}$, and $f^{\prime}\left(x^{*}\right)=-1$. Then $x^{*}$ is either stable or unstable; it cannot be semistable.

This method can be improved, as we will see in the sequel.

3. Another method. One of the drawbacks of the previous algorithm for the case of a fixed point with $f^{\prime}\left(x^{*}\right)=-1$ is the need to pass to $g(x)$. To study an $n$th degree polynomial with coefficients bounded by $N$, we need to consider an $n^{2}$-d degree polynomial with coefficients bounded by $N N^{n}\left(\begin{array}{c}n \\ n / 2\end{array}\right) \sim 2^{n} N^{n+1} / \sqrt{n}$. A test using $f(x)$ would avoid this difficulty.

EXAMPLE 3.1. Consider $f(x)=-x+2 x^{2}-4 x^{3}$. To classify the fixed point $f(0)=0$, we compute the derivatives of $f: f^{\prime}(0)=-1, f^{\prime \prime}(0)=4, f^{\prime \prime \prime}(0)=-24$, and zero from then on. We first find $S f(0)=-f^{\prime \prime \prime}(0)-1.5\left(f^{\prime \prime}(0)\right)^{2}=0$. Unfortunately, this falls in the gap of the classical theory. Therefore, to classify 0 , the previous algorithm requires us to pass to the substantially more complicated $g(x)=f(f(x))=-x+4 x^{2}-8 x^{3}+$ $64 x^{5}-192 x^{6}+384 x^{7}-384 x^{8}+256 x^{9}$, to find that $g^{(5)}(0)=(64)(5 !)=7680>0$, making 0 an unstable fixed point.

An improvement to the algorithm is made possible by a formula published in the mid-nineteenth century by Faà di Bruno. For a history of this result as well as some biographical information, see [6].

THEOREM 3.2 (Faà di Bruno).

$$
\frac{d^{n}}{d x^{n}} f(f(x))=\sum \frac{n !}{a_{1} ! a_{2} ! \cdots a_{n} !} f^{(a)}(f(x))\left(\frac{f^{\prime}(x)}{1 !}\right)^{a_{1}} \cdots\left(\frac{f^{(n)}(x)}{n !}\right)^{a_{n}}
$$

for $f \in \mathbb{C}^{n}$ and where $a=a_{1}+a_{2}+\cdots+a_{n}$ and the sum extends over all possible integer $a_{i}$ such that $0 \leq a_{i} \leq n$ and $n=a_{1}+2 a_{2}+3 a_{3}+\cdots+n a_{n}$.

In our context, we are evaluating it all at the fixed point $x^{*}$, with $g(x)=f(f(x))$ and $f^{\prime}\left(x^{*}\right)=-1$. The sum then becomes

$$
g^{(n)}\left(x^{*}\right)=\sum \frac{(-1)^{a_{1}} n ! f^{(a)}\left(x^{*}\right)}{a_{1} ! a_{2} ! \cdots a_{n} !}\left(\frac{f^{(2)}\left(x^{*}\right)}{2 !}\right)^{a_{2}} \cdots\left(\frac{f^{(n)}\left(x^{*}\right)}{n !}\right)^{a_{n}} .
$$

The character of this result will be more evident with several examples. 
EXAMPLE 3.3. For $n=2$, there are two summands: $\left\{a_{1}=2, a_{2}=0\right\}$ and $\left\{a_{1}=\right.$ $0, a_{2}=1$ \}. Hence $g^{(2)}\left(x^{*}\right)=\left((-1)^{2} 2 ! / 2 !\right) f^{\prime \prime}\left(x^{*}\right)+\left((-1)^{0} 2 ! / 1 !\right) f^{\prime}\left(x^{*}\right)\left(f^{\prime \prime}\left(x^{*}\right) / 2 !\right)=$ $f^{\prime \prime}\left(x^{*}\right)-f^{\prime \prime}\left(x^{*}\right)=0$. This is no coincidence; Theorem 2.3 does not allow the first nonzero derivative of $g$ to be even-numbered.

For $n=3$, there are three terms: $\left\{a_{1}=3, a_{2}=a_{3}=0\right\},\left\{a_{1}=a_{2}=1, a_{3}=0\right\}$, and $\left\{a_{1}=\right.$ $\left.a_{2}=0, a_{3}=1\right\}$. Hence $g^{(3)}\left(x^{*}\right)=\left((-1)^{3} 3 ! / 3 !\right) f^{(3)}\left(x^{*}\right)+\left((-1)^{1} 3 ! / 1 ! 1 !\right) f^{\prime \prime}\left(x^{*}\right)\left(f^{\prime \prime}\left(x^{*}\right) /\right.$ $2 !)+\left((-1)^{0} 3 ! / 1 !\right) f^{\prime}\left(x^{*}\right)\left(f^{(3)}\left(x^{*}\right) / 3 !\right)=-f^{(3)}\left(x^{*}\right)-3\left(f^{\prime \prime}\left(x^{*}\right)\right)^{2}-f^{(3)}\left(x^{*}\right)=2 S f\left(x^{*}\right)$.

This confirms the classical test.

4. Generalized Schwarzian-type derivatives. Using formula (3.2), we can calculate generalized analogues of the Schwarzian derivative to use in our classification. We take $S_{k} f(x)=(1 / 2) g^{(2 k+1)}(x)$, and simplify using the assumption that $S_{i} f(x)=0$ for all $i<k$ :

$$
\begin{aligned}
S_{1} f(x)= & S f(x)=-f^{(3)}(x)-\frac{3}{2}\left(f^{\prime \prime}(x)\right)^{2}, \\
S_{2} f(x)= & -f^{(5)}(x)-\frac{15}{2} f^{\prime \prime}(x) f^{(4)}(x)+15\left(f^{\prime \prime}(x)\right)^{4}, \\
S_{3} f(x)= & -f^{(7)}(x)-14 f^{\prime \prime}(x) f^{(6)}(x)+\frac{945}{2}\left(f^{\prime \prime}(x)\right)^{3} f^{(4)}(x) \\
& -\frac{35}{4}\left(f^{(4)}(x)\right)^{2}-\frac{9045}{4}\left(f^{\prime \prime}(x)\right)^{6}, \\
S_{4} f(x)= & -f^{(9)}(x)-\frac{45}{2} f^{\prime \prime}(x) f^{(8)}(x)+2205\left(f^{\prime \prime}(x)\right)^{3} f^{(6)}(x) \\
& -\frac{208845}{2}\left(f^{\prime \prime}(x)\right)^{5} f^{(4)}(x)+\frac{411075}{2}\left(f^{\prime \prime}(x)\right)^{8} \\
& -105 f^{(4)}(x) f^{(6)}(x)+7875\left(f^{\prime \prime}(x)\right)^{2}\left(f^{(4)}(x)\right)^{2}, \\
S_{5} f(x)= & -f^{(11)}(x)-90748350\left(f^{\prime \prime}(x)\right)^{10}-\frac{495}{2} f^{(4)}(x) f^{(8)}(x) \\
& -\frac{21881475}{4}\left(f^{\prime \prime}(x)\right)^{4}\left(f^{(4)}(x)\right)^{2}+\frac{30195}{4}\left(f^{\prime \prime}(x)\right)^{3} f^{(8)}(x) \\
& +\frac{201611025}{4}\left(f^{\prime \prime}(x)\right)^{7} f^{(4)}(x)+\frac{317625}{4} f^{\prime \prime}(x)\left(f^{(4)}(x)\right)^{3} \\
& -33 f^{\prime \prime}(x) f^{(10)}(x)+\frac{197505}{2}\left(f^{\prime \prime}(x)\right)^{2} f^{(4)}(x) f^{(6)}(x) \\
& -\frac{1943865}{2}\left(f^{\prime \prime}(x)\right)^{5} f^{(6)}(x)-231\left(f^{(6)}(x)\right)^{2} .
\end{aligned}
$$

This allows a simpler algorithm to classify oscillatory nonhyperbolic fixed points of a one-dimensional map.

THEOREM 4.1. Let $f: \mathbb{R} \rightarrow \mathbb{R}$ be continuous with $f\left(x^{*}\right)=x^{*}$ and $f^{\prime}\left(x^{*}\right)=-1$. Let $k \geq 1$ be minimal such that $S_{k} f\left(x^{*}\right)=A \neq 0$. Then $x^{*}$ is classified as follows:

(1) if $A>0$, then $x^{*}$ is unstable,

(2) if $A<0$, then $x^{*}$ is stable.

Theorem 4.1 allows classification of fixed points with simpler calculation. Additional Schwarzian-type derivatives are simple to calculate using formula (3.2). However, in 
general this will not be necessary, as each generalized derivative is only needed if all earlier ones are zero.

EXAMPLE 4.2. Consider again $f(x)=-x+2 x^{2}-4 x^{3}$, and recall that $f^{\prime}(0)=-1$, $f^{\prime \prime}(0)=4$ and $f^{(3)}(0)=-24$. We see that $S_{1} f(0)=-(-24)-(3 / 2)(4)^{2}=0$ and that $S_{2} f(0)=0-0+15(4)^{4}=3840>0$. Hence, 0 is an unstable fixed point of $f(x)$.

EXAMPLE 4.3. Consider the function $f(x)=-x+x^{2}-x^{3}+(2 / 3) x^{4}$. Observe that $f^{\prime}(0)=-1, f^{\prime \prime}(0)=2, f^{(3)}(0)=-6$, and $f^{(4)}(0)=16$. We see that $S_{1} f(0)=-(-6)-$ $(3 / 2)(2)^{2}=0, S_{2} f(0)=-0-(15 / 2)(2)(16)+15(2)^{4}=0$, and $S_{3} f(0)=-0-14(2)(0)+$ $(945 / 2)(2)^{3}(16)-(35 / 4)(16)^{2}-(9045 / 4)(2)^{6}=-86480<0$. Hence, 0 is a stable fixed point of $f(x)$.

Several properties of these $S_{k} f(x)$ are described in the following result.

THEOREM 4.4. $S_{k} f(x)$ has exactly one term containing an odd derivative of $f$, and that term is $-f^{(2 k+1)}(x)$.

Proof. First, recall that $S_{k} f(x)=(1 / 2) g^{(2 k+1)}(x)$. We now use formula (3.2) with $n=2 k+1$. Observe that the highest derivative that can appear is $f^{(n)}\left(x^{*}\right)$. This can appear in a term in only two ways: if $a=n$ or if $a_{n}>0$. The restrictions on the sum force exactly two terms containing $f^{(n)}\left(x^{*}\right):\left\{a_{1}=n, a_{2}=\cdots=a_{n}=0\right\}$ and $\left\{a_{1}=\right.$ $\left.\cdots=a_{n-1}=0, a_{n}=1\right\}$. Each of these terms simplifies to $-f^{(2 k+1)}\left(x^{*}\right)$. Hence, $S_{k} f(x)$ has the required term $-f^{(2 k+1)}(x)$.

Now, we prove by strong induction that no other terms appear with odd derivatives of $f$. The case $k=1$ corresponds to the classical Schwarzian derivative. For $k>1$, we observe that we are simplifying under the assumption that $S_{i} f(x)=0$ for all $i<k$. The result holds for these $S_{i} f(x)$ by the induction hypothesis. Hence, we can solve for each odd derivative $f^{(2 i+1)}(x)$ in terms of even derivatives, and substitute into $S_{k} f(x)$.

We now use these Schwarzian-type derivatives to generate a class of functions, each of which is analytically a "square root" of the identity at the origin.

5. A special class of functions. The function $h(x)=x$ has a natural square root, namely, $f(x)=-x$. By this we mean that $f(f(x))=h(x)=x$. However, we can construct an infinite class of other functions $f$, each of which is analytically a square root of $h(x)$ at the origin. That is, $(f \circ f)^{(n)}(0)=h^{(n)}(0)$ for all $n \geq 0$.

The most general square root is given by the following power series:

$$
f(x)=-x+\frac{a_{2}}{2 !} x^{2}+\frac{a_{3}}{3 !} x^{3}+\frac{a_{4}}{4 !} x^{4}+\cdots+\frac{a_{n}}{n !} x^{n}+\cdots
$$

We observe that $f(0)=0, f^{\prime}(0)=-1$, and otherwise $f^{(n)}(x)=a_{n}$. In order to ensure that $(f \circ f)^{(n)}(0)=h^{(n)}(0)=0$, we must ensure that $S f_{k}(0)=0$ for each $k$. We can do this inductively by choosing the odd derivatives $\left(a_{2 j+1}\right)$ as per Theorem 4.4. The even derivatives $\left(a_{2 j}\right)$ may be chosen freely. For example, we may choose $a_{2}$ freely, but then $a_{3}=-(3 / 2) a_{2}^{2}$ to have $S_{1} f(x)=0$. 
If we choose the even coefficients growing not too fast, the odd coefficients will also grow not too fast, by the following result.

THEOREM 5.1. Suppose that $\left|a_{i}\right| \leq 1$ for $2 \leq i \leq n$. Then (assuming $n$ is sufficiently large), $\left|a_{n} / n !\right| \leq 1$.

Proof. In formula (3.2), there is exactly one term for each partition of $n$. Hence, there are at most $e^{\pi \sqrt{2 n / 3}}$ terms (for a proof of this bound, see [13]).

We will now show that each term has a coefficient bounded above by $n ! / 2^{\lfloor n / 2\rfloor}$. If any of $a_{\lfloor n / 2\rfloor+1}, \ldots, a_{n}$ is positive, then the coefficient of that term is at most $n ! /(\lfloor n / 2\rfloor+$ $1) !<n ! / 2^{\lfloor n / 2\rfloor}$ for $n \geq 4$. Otherwise, we have $0=a_{\lfloor n / 2\rfloor+1}=a_{\lfloor n / 2\rfloor+2}=\cdots=a_{n}$. In this case, we have $a_{1}+a_{2}+\cdots+a_{\lfloor n / 2\rfloor}=n$, and the coefficient is at most $n ! / a_{1} ! a_{2} ! \cdots a_{\lfloor n / 2\rfloor} !$. This is maximized when $2=a_{1}=a_{2}=\cdots=a_{\lfloor n / 2\rfloor}$. Hence, in any case, the coefficient of each term is bounded above by $n ! / 2^{\lfloor n / 2\rfloor}$.

So, putting together the two bounds above with the hypothesis that each derivative $\left|f^{(i)}\left(x^{*}\right)\right| \leq 1$, we get $\left|a_{n} / n !\right| \leq e^{\pi \sqrt{2 n / 3}} / 2^{\lfloor n / 2\rfloor} \leq e^{\pi \sqrt{2 / 3} \sqrt{n}-(\ln 2 / 2) n} \leq 1$ for $n$ sufficiently large (it turns out that $n \geq 55$ is sufficient).

This bound is not sharp. The terms of formula (3.2) are of both signs, which is not exploited by this result. Also, the lower derivatives appear as multiple powers, and so there could be a further improvement that way, if we insist they are strictly less than one in absolute value. Calculation of polynomial approximations to these power series suggests that they can be made to have a positive radius of convergence, but this is unproven.

6. Open problems. This work has created more questions than it has answered.

CONJECTURE 6.1. Given $M>0$, there are infinitely many square roots of the identity that converge on $[-M, M]$.

CONJECTURE 6.2. The terms of $S_{k} f(x)$, except for the unique highest-derivative term, are all of the form $\alpha\left(f^{\left(a_{1}\right)}\right)^{b_{1}} \cdots\left(f^{\left(a_{j}\right)}\right)^{b_{j}}$, where $4 \alpha \in \mathbb{Z}$ and $2 n=\sum_{i=1}^{j} b_{i}\left(a_{i}-1\right)$.

Problem 6.3. Find a combinatorial proof that if $f^{\prime}\left(x^{*}\right)=-1$ and $g^{\prime \prime}\left(x^{*}\right)=\cdots=$ $g^{(2 k-1)}\left(x^{*}\right)=0$, then $g^{(2 k)}\left(x^{*}\right)=0$.

Problem 6.4. Find a simple expression for the coefficients of $S_{k} f(x)$.

\section{REFERENCES}

[1] K. T. Alligood, T. D. Sauer, and J. A. Yorke, Chaos, Textbooks in Mathematical Sciences, Springer-Verlag, New York, 1997.

[2] F. M. Dannan, S. N. Elaydi, and V. Ponomarenko, Stability of hyperbolic and nonhyperbolic fixed points of one-dimensional maps, J. Difference Equ. Appl. 9 (2003), no. 5, 449457.

[3] R. L. Devaney, An Introduction to Chaotic Dynamical Systems, 2nd ed., Addison-Wesley Studies in Nonlinearity, Addison-Wesley Publishing Company Advanced Book Program, California, 1989.

[4] S. N. Elaydi, An Introduction to Difference Equations, 2nd ed., Undergraduate Texts in Mathematics, Springer-Verlag, New York, 1999. 
[5] Discrete Chaos, Chapman \& Hall/CRC, Florida, 2000.

[6] W. P. Johnson, The curious history of Faà di Bruno's formula, Amer. Math. Monthly 109 (2002), no. 3, 217-234.

[7] W. G. Kelley and A. C. Peterson, Difference Equations, 2nd ed., Harcourt/Academic Press, California, 2001.

[8] E. Lorenz, Deterministic nonperiodic flow, J. Atmospheric Sci. 20 (1963), 130-141.

[9] R. M. May, Biological populations obeying difference equations: stable points, stable cycles and chaos, Biol. Cybernet 42 (1982), 221-229.

[10] R. E. Mickens, Difference Equations, 2nd ed., Van Nostrand Reinhold, New York, 1990.

[11] R. K. Miller and A. N. Michel, Ordinary Differential Equations, Academic Press, New York, 1982.

[12] C. Robinson, Dynamical Systems, 2nd ed., Studies in Advanced Mathematics, CRC Press, Florida, 1999.

[13] J. H. van Lint and R. M. Wilson, A Course in Combinatorics, Cambridge University Press, Cambridge, 1992.

Vadim Ponomarenko: Department of Mathematics, Trinity University, 715 Stadium Drive, San Antonio, TX 78212-7200, USA

E-mail address: vadim@trinity.edu 


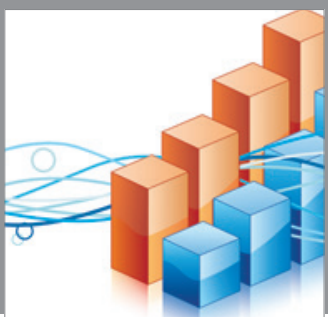

Advances in

Operations Research

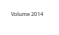

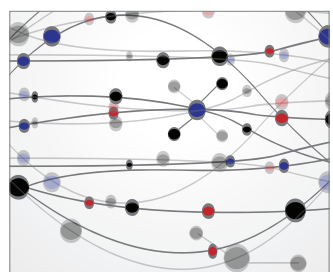

\section{The Scientific} World Journal
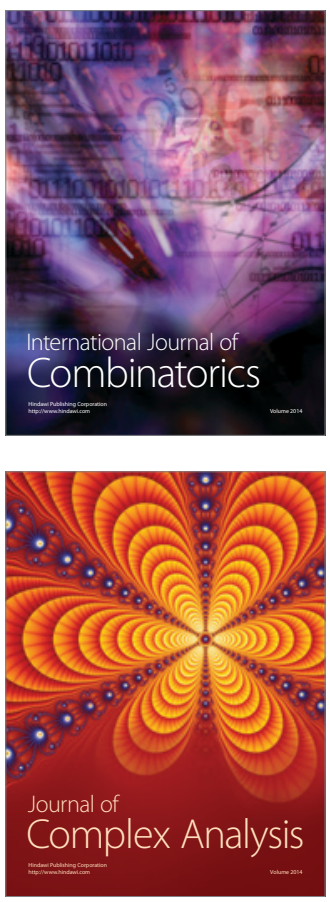

International Journal of

Mathematics and

Mathematical

Sciences
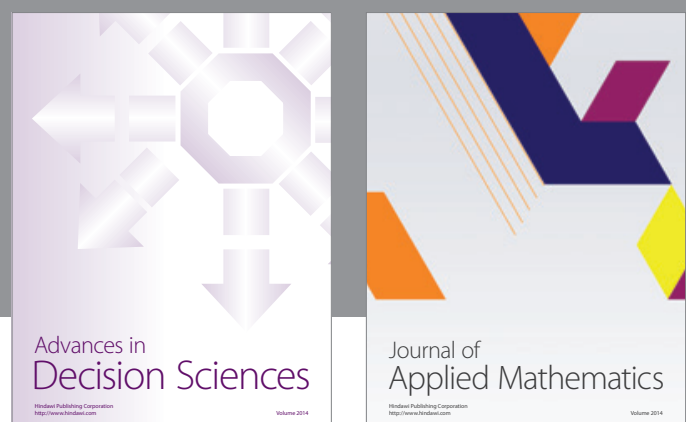

Journal of

Applied Mathematics
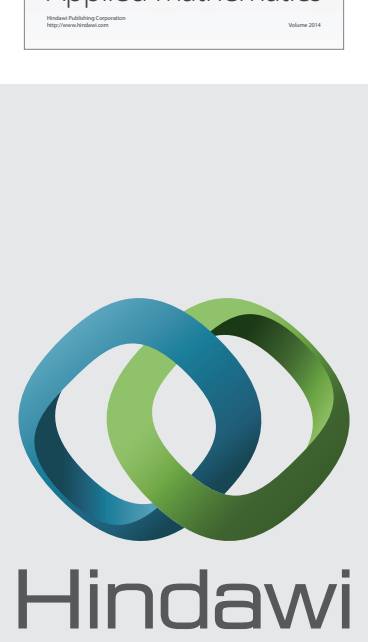

Submit your manuscripts at http://www.hindawi.com
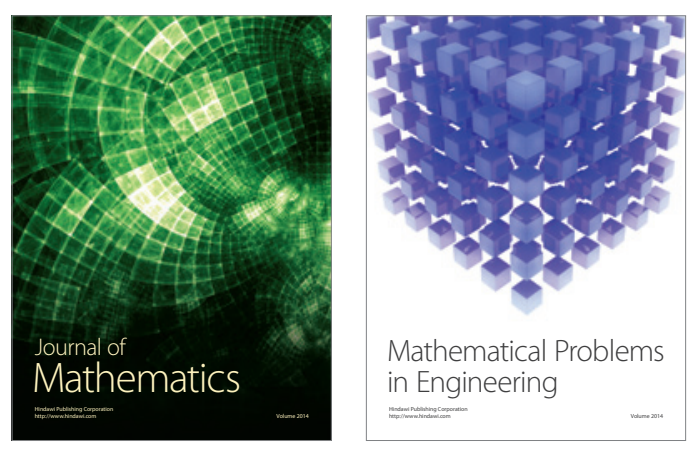

Mathematical Problems in Engineering
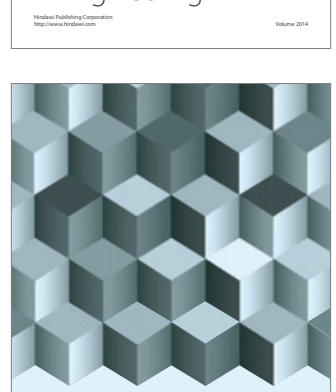

Journal of

Function Spaces
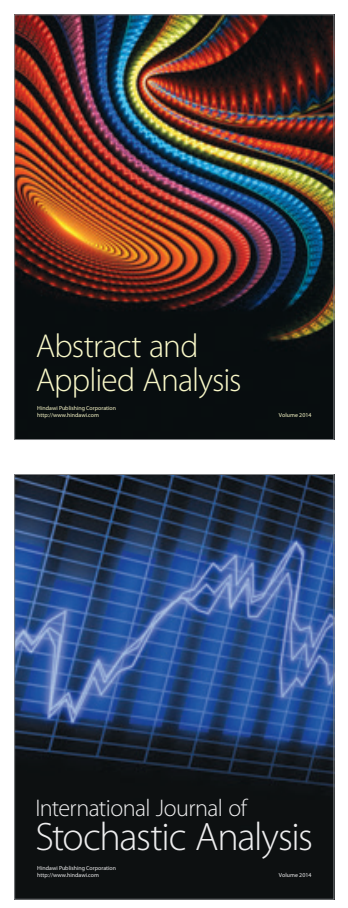

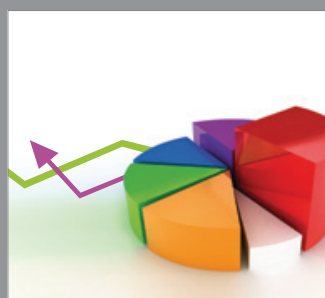

ournal of

Probability and Statistics

Promensencen
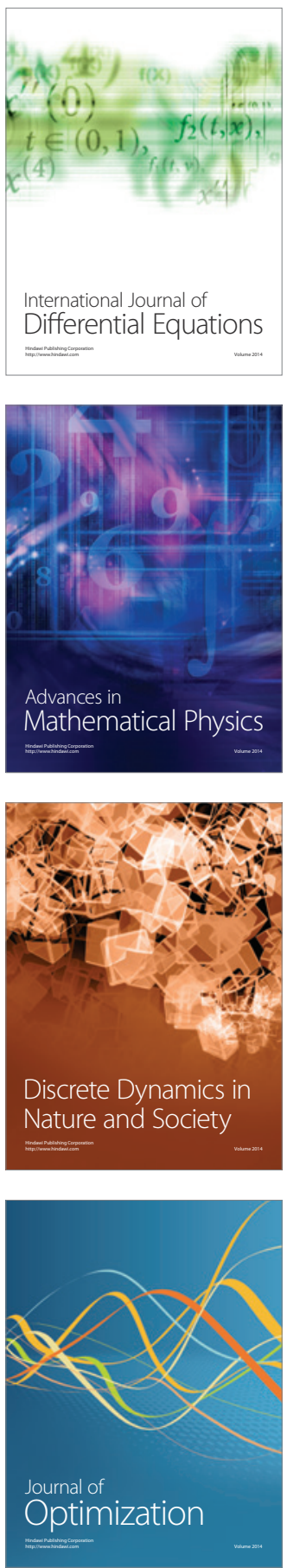